\title{
AR Navigation System for Neurosurgery
}

\author{
Yuichiro Akatsuka $^{2)}$, Takakazu Kawamata ${ }^{1)}$, Masakazu Fujii ${ }^{2)}$,Yukihito \\ Furuhashi $^{2)}$, Akito Saito ${ }^{2)}$, Takao Shibasaki ${ }^{2}$, Hiroshi Iseki ${ }^{1)}$, and Tomokatsu Hori ${ }^{1)}$ \\ ${ }^{1)}$ Dept. of Neurosurgery, Neurological Institute, Tokyo Women's Medical University \\ ${ }^{2}$ Advanced Technology Research Center, Olympus Optical Co., Ltd. \\ Address: 2-3 Kuboyama-cho Hachioji-shi Tokyo, 192-8512 Japan \\ Email: y_akatsuka@ot.olympus.co.jp
}

\begin{abstract}
This paper presents a navigation system for an endoscope which can be used for neurosurgery. In this system, a wire frame model of a target tumor and other significant anatomical landmarks are superimposed in real-time onto live video images taken from the endoscope. The wire frame model is generated from a CT/MRI slice images. Overlaid images are simultaneously displayed in the same monitor using the picture-in-picture function so that the surgeon can concentrate on the single monitor during the surgery. The system measures the position and orientation of the patient using specially designed non-contact sensing devices mounted on the endoscpe. Based on this real-time measurement, the system displays other useful information about the navigation as well as the rendered wire frame. The accuracy of registration between the wire frame model and the actual live view is less than $2 \mathrm{~mm}$. We applied this AR navigation clinically in surgical resection of pituitary tumors in six cases, and verified its performance and effectiveness.
\end{abstract}

\section{Introduction}

Recently, the minimally invasive surgery has been playing a critical role over in the field of neurosurgery. Several attempts have been already made for this fields [1][2]. The surgeons need to carefully observe two or more different scopes and/or monitors to acquire visual information about the operational status. A prospective surgical navigator needs to provide efficient and effective navigational components as a good human interface function, as well as to provide the state-of-the-art technologies of sensing and control devices.

We developed an augmented reality (AR) navigation system especially suitable for neurosurgery[3]. This system helps the surgeons to navigate an endoscopes to the target in the patient body, while displaying efficient and effective visual information about the relative position to the target and the instrument(s) as well as surgical plans on a single monitor. First, 3D wire frame models of target tumor and other significant anatomical landmarks are generated from patient's CT/MRI slices images and are registered with the patient body coordinate frame. During the surgery, the relative position and orientation of the patient body and the surgical instruments are measured in real-time, and a wire frame model scene of the target tumor is generated and superimposed onto the live video images taken from the endoscope so that the operators can acquire navigational information. 
In this paper, we will first present the system architecture and then usefulness and effectiveness of the system by showing the result of clinical test.

\section{System}

The system consists of an endoscope (Olympus), an optical tracking system, and a controller. The optical tracking system (I.G.T. Flashpoint $5000-3 \mathrm{D}$ localizer) uses two sets of infrared LEDs to measure the position and the orientation of the endoscope respect to the patient body.

The first set of LEDs is attached on the head of the patient to measure the position and orientation of the patient head with respect to the reference frame. The second set of LEDs (shown in Fig.1 with cyan circle) is mounted on the endoscope to measure the position and orientation of the endoscope with respect to the reference frame. By taking into account the above two sets of measurements, the system calculates the relative position and orientation of the patient body and the endoscope so that the patient movement can be allowed during the surgery.

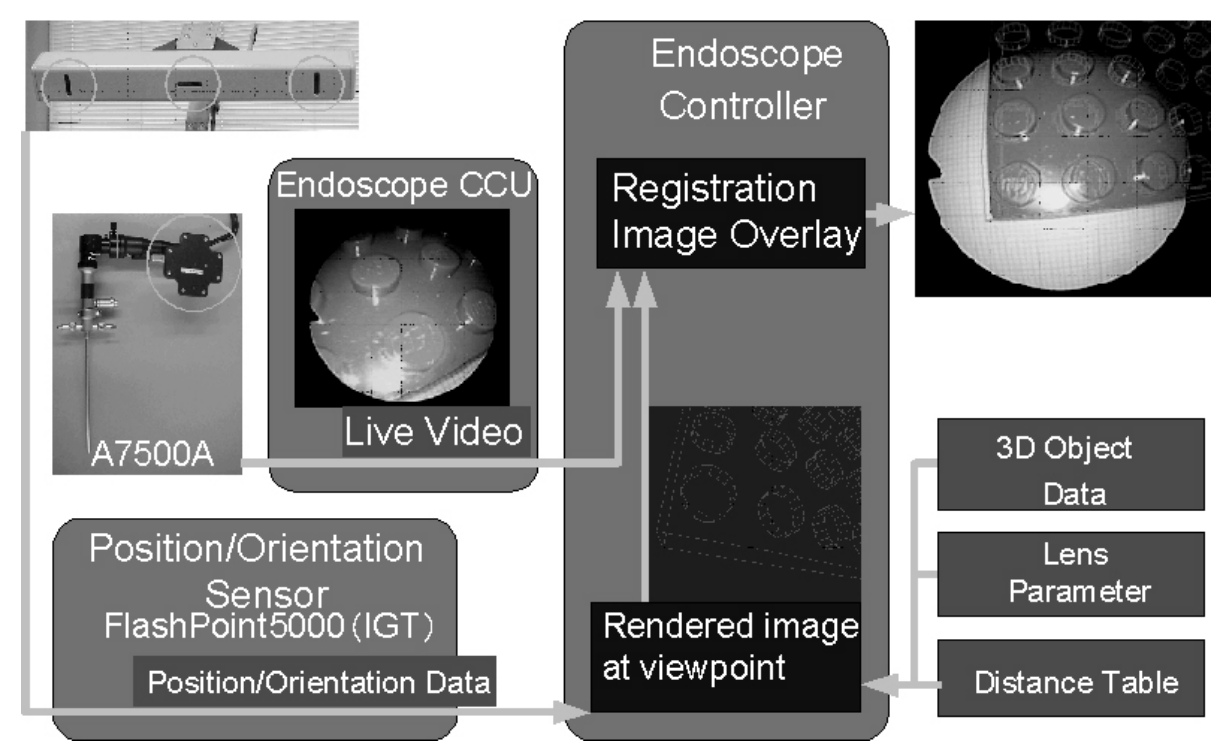

Fig. 1. AR Navigation system

The controller (Pentium III PC) generates the wire frame model scenes of the tumor and other significant anatomical landmarks with the measured position and orientation of the endoscope as a viewpoint. The $3 \mathrm{D}$ wire frame models were previously reconstructed from patient's MRI data and superimposed to the scenes onto the endoscopic live images. 
One good feature here is that the system takes into account the lens distortion observed in the endoscopic views. More specifically, the wire frame model scene is adjusted based on the lens distortion coefficient. Another good feature associated with the endoscope is that the system extends the wire frame scene beyond limited circular endoscopic views so that the surgeons can visually estimate the shape of the tumor beyond the viewing area.

In prior to the operation, the tumor and significant anatomical landmarks were reconstructed as a 3D wire frame model from patient's MRI data. On the monitor, three slice views of MRI, axial, coronal, and sagittal views, are displayed at the corners, and the position and the orientation of the endoscope are shown over slice views.

The colors of the wire frame images are controlled based on the tip position of the endoscope with respect to the target tumor. The tumor is colored "blue" in Fig.2 and "yellow" in Fig.3. This describes that the distance between the tumor and the tip position of the endoscope is less than $10 \mathrm{~mm}$ in the case of Fig.3. The distance is also shown in the bar graph and figures at the top of the screen. we verified that the registration accuracy of the image overlay is less than $2 \mathrm{~mm}[3]$.

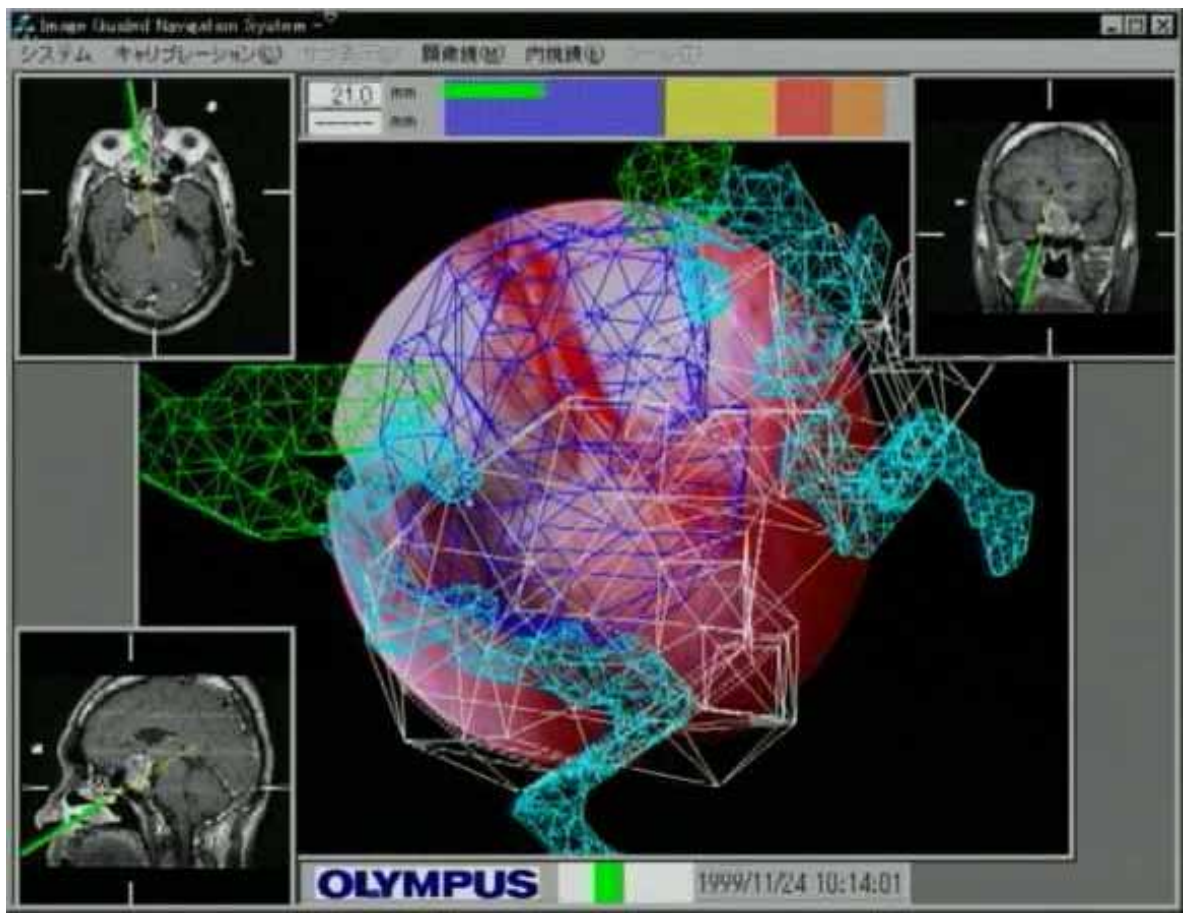

Fig. 2. Endoscopic view during surgery for pituitary tumor with superimposed images. The anterior wall of the sphenoid sinus is approached. The green, blue, light blue, and white wire frame images indicate optic nerves, tumor, internal carotid arteries, and sphenoid sinus, respectively. The upper color bar indicates reaching point of the endoscope toward the tumor. Three MRI images (axial, coronal, and sagittal) are shown on the monitor with indication of endoscopic direction (green) andendoscopic beam (yellow). 


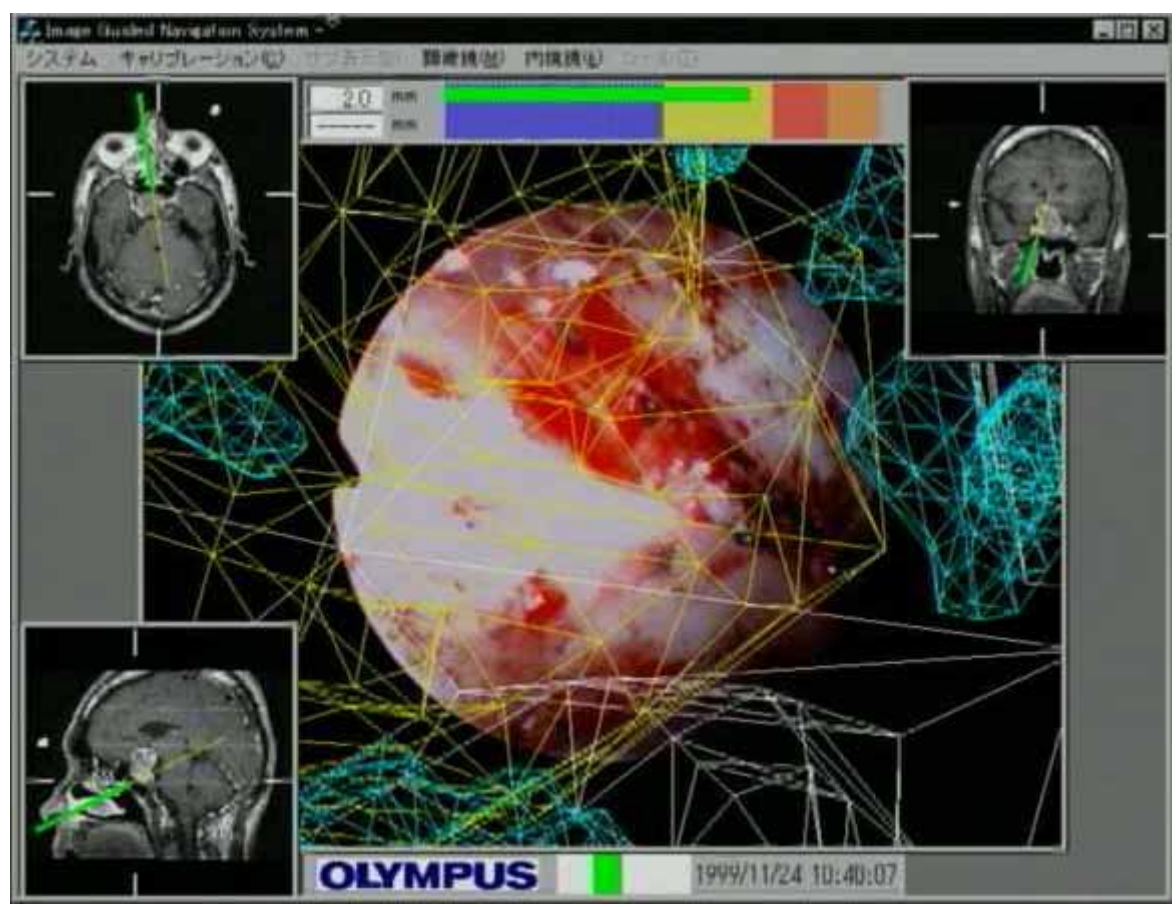

Fig. 3. Endoscopic view during surgery for pituitary tumor with superimposed images. The basal face of the sellar floor is approached following anterior sphenoidotomy. The yellow, light blue, and white wire frame images indicate tumor, internal carotid arteries, and sphenoid sinus, respectively. The upper color bar indicates reaching point of the endoscope toward the tumor. Three MRI images (axial, coronal, and sagittal) are shown on the monitor with indication of endoscopic direction (green) and endoscopic beam (yellow).

\section{Clinical Test}

We applied the AR navigation clinically in surgical resection of pituitary tumors in six cases. The patients underwent endoscope-assisted unilateral endonasal transsphenoidal surgery. Wire frame images of the anatomical structures of sphenoid sinuses, optic nerves, and internal carotid arteries, and tumors were superimposed onto the live images taken from the endoscope (Figure 2, 3). The AR navigation system indicated location of the tumors and surrounding anatomical structures precisely. We could detect the location of the tumor easily. One of the most important points for safe surgery in the endonasal transsphenoidal approach is making sure the midline. The midline was always identified in the AR navigation system when we used an endoscope. Furthermore, location and direction of an endoscope itself was also indicated on MRI images on the monitor (green) with direction of endoscopic beam (yellow) (Figure 2, 3). This was very useful because neurosurgeons are sometimes confused at the direction observed in an endoscope with 30 or 70 degrees. Intraoperative scene is demonstrated in Figure 4. 


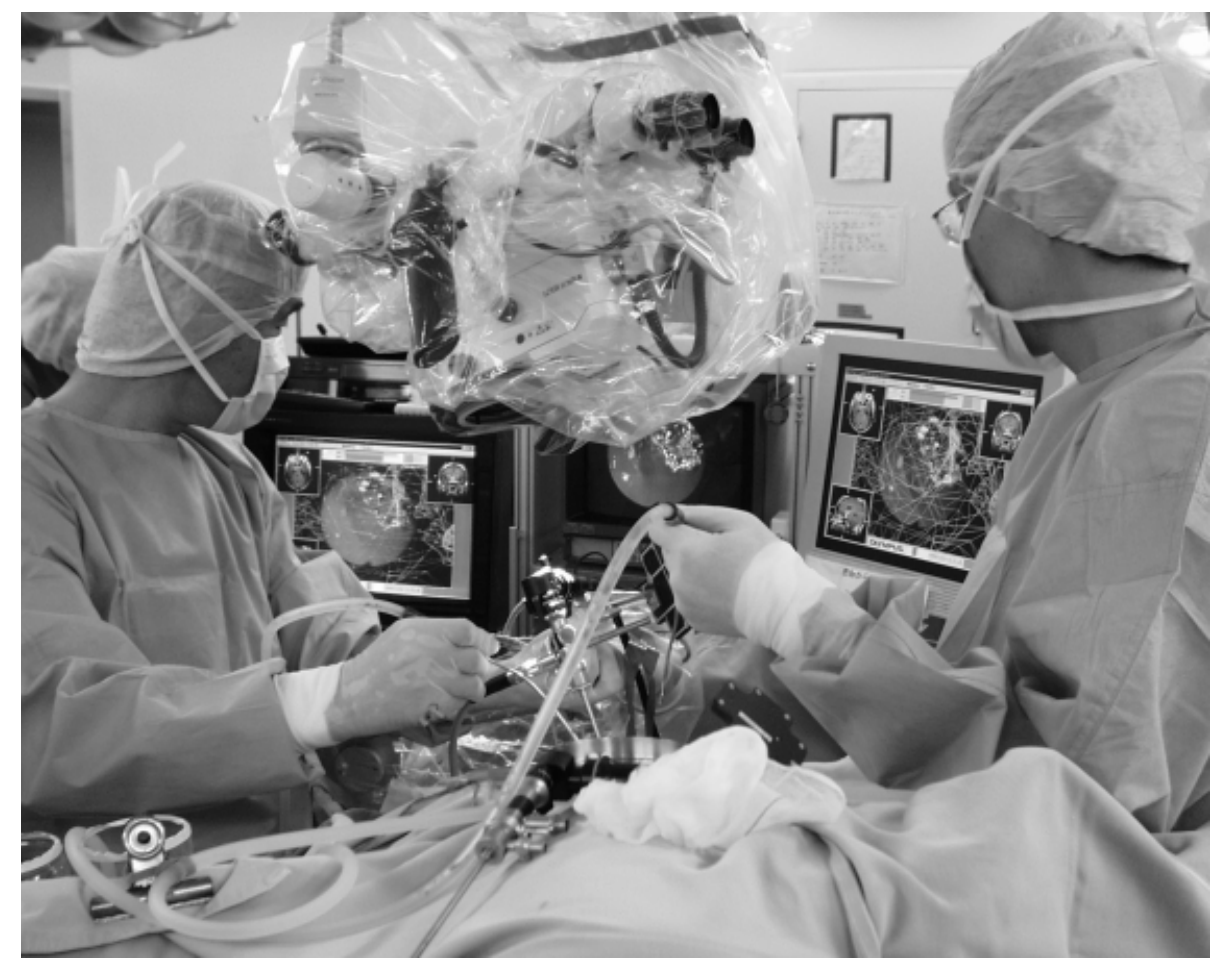

Fig. 4. Intraoperative scene of pituitary tumor surgery. Our endonasal transsphenoidal surgery is performed using both a microsurgery and an endoscope. Monitors of endoscopic live image and AR navigation are set up.

\section{Conclusions and Future Works}

The AR navigation we developed was very efficient in the endonasal transsphenoidal surgery for pituitary tumors in detecting location of tumors and surrounding anatomical structures. It was also effective to perform safe surgeries.

For further clinical application of the AR navigation system in pituitary tumor surgeries, it may be necessary to ensure the more absolute accuracy. Furthermore, it is better to set up the AR navigation system easily preoperatively.

\section{Acknowledgements}

This work was supported in part by Information-technology Promotion Agency, Japan. 


\section{References}

[1] T. Dohi : Computer SurgeryandMedical Image Processing, Medical Imaging Technology Vol.12 No.5 (1994) pp 612-618

[2] H. Iseki et. al. : Medical science and virtual reality - Computer aided surgery (CAS)-, Proceedings of 73th Annual Meeting (The Japan Society of Mechanical Engineers) (1996) Narashino

[3] Y. Akatsuka et. al. :Navigation System for Neurosergery with PC Platform, Medicine Meets Virtual Reality 2000 Proceedings pp10-16(2000) Newport Beach, CA 